\title{
Bite of the wolf: innate immune responses propagate autoimmunity in lupus
}

\author{
Sarthak Gupta and Mariana J. Kaplan \\ Systemic Autoimmunity Branch, Intramural Research Program, National Institute of Arthritis and Musculoskeletal and Skin Diseases (NIAMS), NIH, Bethesda, Maryland, USA.
}

\begin{abstract}
The etiopathogenesis of systemic lupus erythematosus (SLE), a clinically heterogeneous multisystemic syndrome that derives its name from the initial characterization of facial lesions that resemble the bite of a wolf, is considered a complex, multifactorial interplay between underlying genetic susceptibility factors and the environment. Prominent pathogenic factors include the induction of aberrant cell death pathways coupled with defective cell death clearance mechanisms that promote excessive externalization of modified cellular and nuclear debris with subsequent loss of tolerance to a wide variety of autoantigens and innate and adaptive immune dysregulation. While abnormalities in adaptive immunity are well recognized and are key to the pathogenesis of SLE, recent findings have emphasized fundamental roles of the innate immune system in the initiation and propagation of autoimmunity and the development of organ damage in this disease. This Review focuses on recent discoveries regarding the role of components of the innate immune system, specifically neutrophils and interferons, in promoting various aspects of lupus pathogenesis, with potential implications for novel therapeutic strategies.
\end{abstract}

\section{Introduction}

Systemic lupus erythematosus (SLE) is an autoimmune syndrome of unclear etiology that predominantly affects women and disproportionately afflicts minorities (1). Lupus derives its name from the Latin word for wolf, and early descriptions of this disease used the term to describe the facial lesions that look like a wolf's bite. SLE has pleiotropic clinical manifestations and profound clinical heterogeneity, making its diagnosis and treatment very challenging. Indeed, what we call SLE may be driven by heterogeneous pathways of immune dysregulation that eventually converge into a loosely shared clinical phenotype. SLE affects many organ systems, including skin, kidneys, brain, and the vasculature. Characteristically, SLE presents with periods of clinical and serologic flare interspersed among phases of clinical remission, which can occur spontaneously or modulated by exposure to environmental stimuli, such as ultraviolet light or infections. Recent advances in immunomodulatory/immunosuppressive treatments have improved morbidity and mortality in SLE. However, as patients live longer, prolonged inflammation and exposure to enhanced oxidative stress promote the development of chronic complications, including increased incidence of premature cardiovascular disease (CVD) (2). Furthermore, the ability to predict which patients will respond to specific medications or develop end-organ complications remains an area of need to optimize SLE outcomes.

SLE develops in a multistep process in which genetic, epigenetic, and environmental factors can promote aberrant cell death and ineffective clearance of dead cell debris (3). In genetically

Conflict of interest: The NIAMS has collaborative research agreements with Medlmmune/AstraZeneca, Pfizer, and Bristol Myers Squibb. MJK is on the scientific advisory boards of Neutrolis, Inc. and Cytrill.

Copyright: (c) 2021, American Society for Clinical Investigation.

Reference information: J Clin Invest. 2021;131(3):e144918.

https://doi.org/10.1172/JCl144918. predisposed hosts, this imbalance in dead cell handling can contribute to modification and externalization of nucleic acids and other autoantigens, to promotion of innate and adaptive immune dysregulation, to the development of autoantibodies that predominantly recognize nucleic acids and/or proteins binding to nucleic acids, and to the formation of immune complexes (ICs) that deposit in various organs and promote damage (3). Autoantibody formation can precede clinical disease by many years, suggesting that immune dysregulation is an early event in disease pathogenesis (4). Notably, aspects of immune dysregulation characteristic of SLE have been described in unaffected relatives (5), further supporting a role for both genetic and environmental factors in susceptibility to autoimmunity.

The innate and adaptive arms of the immune system are altered and almost every immune cell type becomes dysregulated in SLE and its various murine models $(3,6)$. The role of the adaptive immune system in SLE has been thoroughly studied and cannot be overemphasized, and various current therapeutic strategies target these immune responses (7). In contrast, the role of various components of the innate immune system in SLE pathogenesis is less well characterized, but recent work emphasizes their crucial role in initiating and perpetuating autoimmunity in this disease. Abnormalities in phenotype and function of monocytes, macrophages $(8,9)$, myeloid and plasmacytoid DCs $(10,11)$, basophils (12), neutrophils (13), NK cells, and $\gamma \delta$ T cells (6) have been described in SLE. Furthermore, dysregulation of fundamental innate immune strategies, such as the complement pathway, synthesis of and response to IFNs, and mechanisms of neural regulation of immune responses, contributes to autoimmunity and tissue damage in SLE $(3,14-16)$. In addition, genetic variations that alter various components of innate immune responses have been reported to contribute to the risk and/or severity of SLE (Table 1).

This Review focuses on the putative roles that the crosstalk between aberrant cell death (particularly the formation of neutro- 
Table 1. Genes that modulate the innate immune system and are associated with SLE risk

\begin{tabular}{|c|c|c|c|}
\hline Gene & Name & Putative mechanism & References \\
\hline FCGR $(2 A, 3 A, 3 B)$ & Fc $\gamma$ receptor & Antibody and immune complex signaling & 132,199 \\
\hline IFIH1 (MDA5) & $\begin{array}{l}\text { Interferon induced with helicase C domain } 1 \text { (encoding melanoma } \\
\text { differentiation-associated protein 5) }\end{array}$ & RNA sensing, IFN signaling pathway & 134 \\
\hline TNFAIP3 (A20) & Tumor necrosis factor- $\alpha$-induced protein 3 & $\begin{array}{c}\text { Disruption of protein's deubiquitinase activity, increased NET formation } \\
\text { through PAD4 modulation }\end{array}$ & 104 \\
\hline PTPN22 & Protein tyrosine phosphatase, nonreceptor type 22 & Neutrophil activation, enhanced NET formation through PAD4 activation & 3,132 \\
\hline TREX1 & Three prime repair exonuclease 1 & Defective nucleic acid degradation & 3 \\
\hline IRF5 & Interferon regulatory factor 5 & IFN signaling pathway & 107,132 \\
\hline IRF7 & Interferon regulatory factor 7 & IFN signaling pathway & 132,199 \\
\hline IRF8 & Interferon regulatory factor 8 & IFN signaling pathway & 199 \\
\hline ITGAM & Integrin $\alpha_{M}$ & Defective leukocyte adhesion and migration, enhanced IFN synthesis & 132 \\
\hline TYK2 & Tyrosine-protein kinase 2 & IFN signaling pathway & 132 \\
\hline TLR9 & Toll-like receptor 9 & Endosomal TLR, DNA sensing, IFN signaling pathway & 199 \\
\hline
\end{tabular}

phil extracellular traps), dysregulation of neutrophils, and the IFN pathway plays in the pathogenesis of SLE and its associated complications, in the context of current and potential therapeutic targets.

\section{SLE and the aberrant handling of cell death}

Dysregulation in cell death pathways, defective clearance of dying cells and their products, and aberrant response to this cell debris by various immune cell subsets are considered fundamental aspects of SLE pathogenesis (Figure 1). This process has been recently reviewed in detail (17). Cell death is associated with modification and redistribution to the cell surface and extracellular space of many ubiquitously expressed autoantigens, which may affect immunologic self-tolerance. Dysregulation in many forms of cell death, including apoptosis, necrosis, necroptosis, pyroptosis, and neutrophil death through formation of neutrophil extracellular traps (NETs), may have diverse effects on immune responses and tissue damage in the context of autoimmunity (17-21). It is important to consider that whether an elicited immune response to the dead cell becomes tolerogenic or immunogenic is determined not only by whether cells die or not, but also by which cells die, and how and where they die (17). A combination of genetic (Table 1), epigenetic, and environmental factors may be involved in driving aberrant cell death, impaired clearance, and/or altered immunologic response to the cell death products, including presentation of modified autoantigens (Figure 1 and refs. 17, 19, 22-25). Various mouse models that fail to clear apoptotic cells develop lupuslike autoimmunity (26-28), and non-phagocytosed dead cells are detected in SLE germinal centers, indicative of impaired clearance (29). These autoantigens may be presented by germinal center follicular DCs to B and T lymphocytes in secondary lymphoid tissues, resulting in loss of tolerance. The formation of ICs containing autoantibodies that recognize nuclear and/or cytoplasmic material released from dead cells can lead to potent downstream inflammatory effects, including the synthesis of type I IFNs that, as discussed below, play prominent roles in SLE pathogenesis (30, 31). In particular, dysregulation in neutrophil cell death and the role of NETs in SLE, as discussed later, display high immunogenic and proinflammatory potential.

\section{Neutrophils, NETs, and SLE pathogenesis}

Neutrophil functions and plasticity. Over the past decade, neutrophils have reemerged as presumed culprits in various aspects of SLE pathogenesis (Figure 1 and ref. 13). As the first responders against invading microorganisms (32), neutrophils develop and terminally differentiate in the bone marrow and are then released into the circulation, where they patrol and sense danger signals $(32,33)$. Under conditions of homeostasis, neutrophils are also found in the lung, spleen, and liver, and they follow tightly regulated circadian rhythms that allow for disarming strategies prior to their migration to various tissues $(33,34)$. Whether disruptions in neutrophils' normal circadian rhythm modulate autoimmune responses remains to be determined. In addition to their antimicrobial roles, neutrophils are responsible for fundamental homeostatic functions, including roles in coagulation, angiogenesis, resolution of inflammation, and tissue repair $(33,35-39)$. Some of these neutrophil properties are induced and modulated by specific tissues, with profound neutrophil intertissue heterogeneity in the steady state (33). Under conditions of injury or infection, neutrophils display their antimicrobial and proinflammatory strategies in tissues $(33,40)$, including the release of neutrophil-derived serine and matrix metalloproteinases (MMPs) that cleave extracellular matrix components, thereby disrupting existing tissue architecture (41). Neutrophils use a complex armamentarium of strategies to immobilize and kill microbes, including phagocytosis and bacterial degradation through synthesis of ROS; release of antimicrobial peptides from granules; and the synthesis of NETs, which are lattices of chromatin bound to granule peptides that have the ability to immobilize and potentially kill various microorganisms (42-44). 


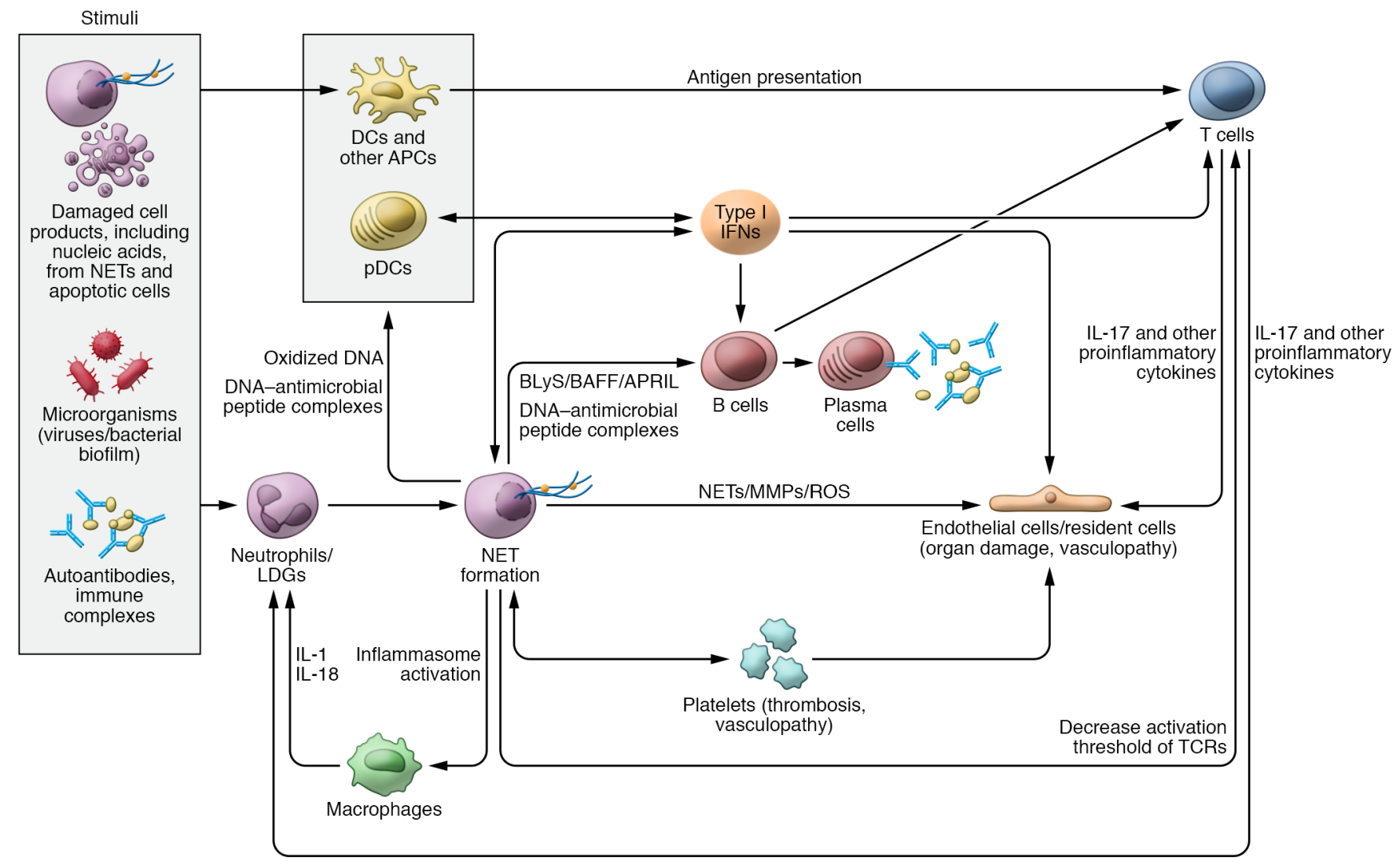

Figure 1. Role of neutrophils, NETs, and IFNs in SLE pathogenesis. Various stimuli can trigger neutrophils to undergo neutrophil extracellular trap (NET) formation. NETs, in turn, can externalize self-antigens, including oxidized DNA and/or DNA-antimicrobial peptide complexes that can be presented to antigen-presenting cells (APCs) and activate plasmacytoid DCs (pDCs) to synthesize type I IFNs. NETs have the ability to activate the NLRP3 inflammasome in macrophages, resulting in increased release of IL-1 and IL-18, which further prime neutrophils to undergo NET formation and perpetuate tissue damage. Different exogenous and endogenous stimuli can promote type I IFN generation. The synthesis of type I IFNs further modulates other APCs, tissue-resident cells, and T and B cell functions. NET products and IFNs modulate T cell responses and can also activate B cells to undergo class switching and secrete autoantibodies against a wide range of self-antigens. DNA-antimicrobial peptide complexes (like LL37-DNA) released from NETs have the ability to directly activate B cells via TLR9 and promote autoantibody generation. NETs directly stimulate T cells by decreasing their activation threshold via Zap70-mediated phosphorylation of the T cell receptor (TCR). Activated T cells release IL-17 and other proinflammatory cytokines that can result in endothelial cell damage as well prime neutrophils to undergo further NET formation and migrate to inflamed tissues. NETs and IFNs can promote direct tissue damage and vascular inflammation through their effect on endothelial cells and platelets. APRIL, a proliferation-inducing ligand; BAFF, B cell activating factor; BLyS, B lymphocyte stimulator; LDG, low-density granulocyte.

NETs are typically generated and released through a distinct programmed inflammatory cell death process (Figure 2 and refs. 13, 43), although mechanisms of NET formation that do not result in cell death have been described $(45,46)$. NETs contain many potent antimicrobial molecules that can stimulate other neutrophils and immune cells $(43,47,48)$. Besides microbial stimuli, NETs are induced by sterile inflammatory stimuli, including platelets $(49,50)$, cytokines (51), uric acid and cholesterol crystals $(52,53)$, various autoantibodies $(54-56)$, and ICs $(55,57,58)$. While the exact mechanisms that lead to NET formation continue to be further refined, this process can occur through several pathways that may or may not involve the NADPH oxidase (NOX) pathway of ROS formation and/or mitochondrial ROS synthesis $(55,59)$. Once initiated, posttranslational modifications of histones including citrullination by the peptidylarginine deiminase (PAD) family of enzymes, particularly PAD4 (60) - promote changes in electrostatic interactions of DNA and histones that disrupt chromatin's structure and promote decondensation of nuclear mate- rial (61). This process is followed by degradation of nuclear and granule membranes and extrusion of NETs into the extracellular space (Figure 2). Gasdermin D, a pore-forming protein considered a key executioner of pyroptosis, has been implicated in certain forms of NET formation $(62,63)$ but not in NETs formed in lupus animal models (64).

The role of aberrant NET formation and clearance in various homeostatic and pathogenic conditions is an area of increased interest. Compared with males, neutrophils from healthy young adult females display enhanced ability to form NETs, both spontaneously and under microbial or sterile inflammatory stimuli (65). This suggests that, in women, neutrophils have a higher propensity to generate and externalize modified autoantigens. In individuals with genetic predisposition to autoimmunity, this enhanced ability to form NETs may contribute to breaking tolerance and may explain, in part, why women are more prone to develop autoimmunity but also mount more robust responses to infectious agents than men. Female neutrophils are also more mature, activated, 


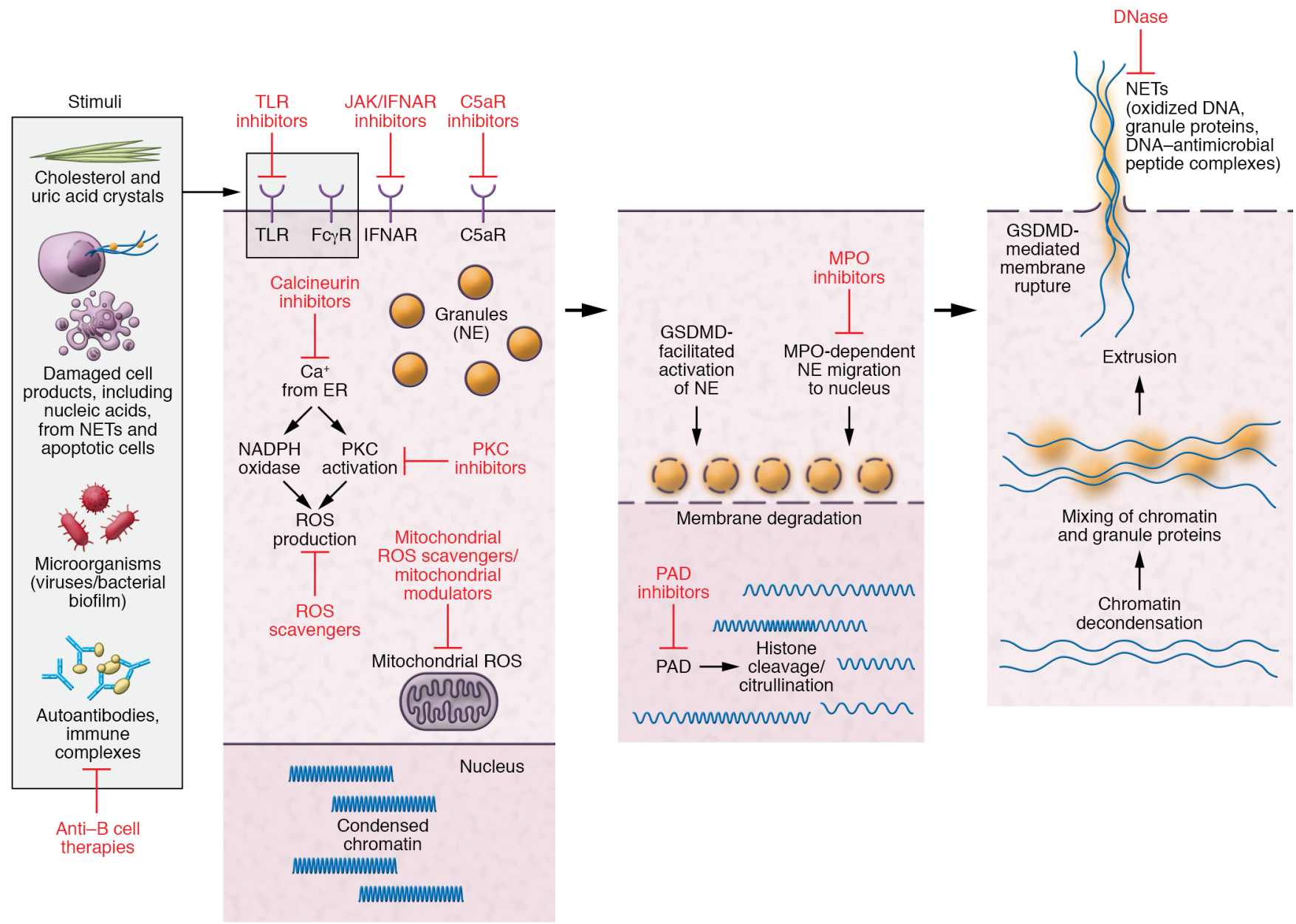

Figure 2. Pathways of NET formation and targets for therapies. Stimulation of neutrophils in an individual genetically predisposed to SLE by various stimuli (microbial, autoantibodies, cell products, etc.) mobilizes calcium from the endoplasmic reticulum that results in activation of protein kinase $\mathrm{C}$ (PKC) and NADPH and/or mitochondrial ROS production. This leads to migration of granule protein to the nucleus and activation of peptidylarginine deiminase-4 (PAD4), which induce citrullination, resulting in changes in electrostatic interactions of DNA and histones, which disrupt chromatin's structure and promote decondensation of nuclear material. This process is followed by degradation of nuclear and granule membranes with mixture of granular protein with chromatin and eventual extrusion of NETs into the extracellular space. Gasdermin D (CSDMD) is implicated in some forms of NET formation, in which ROS-mediated release of neutrophil elastase (NE) processes CSDMD, which further facilitates release and activation of NE. CSDMD also localizes to the plasma membrane to form pores that promote cell lysis to release NETs. Possible therapies targeting critical steps in this pathway (represented by blunt arrows) may be beneficial for SLE. Fc $\gamma$ R, Fc $\gamma$ receptor; IFNAR, type I IFN receptor; JAK, Janus kinases; MPO, myeloperoxidase; TLR, Toll-like receptor.

and readily primed to respond to danger signals than neutrophils from male counterparts, properties that appear to be regulated by sex hormones (66).

Neutrophils regulate other innate and adaptive immune cells in fundamental ways (Figure 1 and refs. 67, 68). These cells can stimulate and suppress $\mathrm{T}$ cell responses in context-dependent manners (69). Various neutrophil granule proteases inhibit $\mathrm{T}$ cell cytokine synthesis, including IL-2 and IL-6 (70). Neutrophils can downregulate the $\zeta$ chain of the $\mathrm{T}$ cell receptor through synthesis of arginase and ROS, promoting T cell arrest (71), and they can express PD-L1 to induce IFN-dependent PD-1-mediated $\mathrm{T}$ cell apoptosis $(72,73)$. Conversely, neutrophils activate $\gamma \delta \mathrm{T}$ cells through cross-presentation of microbial antigens and cross-prime $\mathrm{CD}^{+} \mathrm{T}$ cells in an MHC class I-dependent manner $(74,75)$. Through T cell-independent mechanisms, splenic neutrophils function as B cell helpers (76) and are important sources of B cell activating factor $(\mathrm{BAFF})(77,78)$ and a proliferation-inducing ligand (APRIL) $(79,80)$, molecules that are highly relevant to B cell development and function.

Several neural pathways activated by microbes or endogenous stimuli modulate neutrophil biology (16) through various neuropeptides that inhibit neutrophil function, including calcitonin gene-related peptide and somatostatin (81). Adenosine, a purine nucleoside released by neutrophils at sites of inflammation, has complex effects on neutrophil biology, including NET formation, which depend on the concentration of and signaling through its four receptors (82-84).

Attempts at characterizing neutrophil diversity in recent years have highlighted the presence of neutrophil plasticity and neutrophil subsets based on transcriptional, epigenetic, proteomic, and functional analyses that suggest diverse physiological and pathogenic roles (Table 2 and refs. 39, 85-90). In healthy individuals, several distinct circulating neutrophil subsets were recently identified based on single-cell transcriptomic analysis (66). It remains to 


\section{Table 2. Neutrophil subsets described in the literature}

\begin{tabular}{|c|c|c|c|}
\hline Neutrophil subset & Description & Features & Function \\
\hline $\begin{array}{l}\text { Low-density granulocytes } \\
\text { (LDGs) }\end{array}$ & $\begin{array}{l}\text { Identified in various autoimmune and } \\
\text { autoinflammatory diseases }\end{array}$ & $\begin{array}{l}\text { Colocalize with mononuclear cells upon density } \\
\text { gradient separation }\end{array}$ & $\begin{array}{l}\text { Proinflammatory, with enhanced ability to form } \\
\text { NETs and damage the vasculature }\end{array}$ \\
\hline $\mathrm{LDC}^{\text {mature }}$ & Characterized in SLE & $\begin{array}{l}\text { CD10+; less transcriptionally active; increased } \\
\text { expression of IFN and neutrophil activation genes; } \\
\text { multilobulated nucleus; spontaneous NET formation }\end{array}$ & $\begin{array}{c}\text { Associated with vascular damage and coronary } \\
\text { plaque formation }\end{array}$ \\
\hline LDG immature & Characterized in SLE & $\begin{array}{l}\text { CD10-; transcriptionally active; round/bilobed nucleus; } \\
\text { enhanced degranulation; decreased chemotaxis } \\
\text { and phagocytosis }\end{array}$ & Enhanced degranulation \\
\hline N2 & $\begin{array}{l}\text { Tumor-associated neutrophils seen in majority } \\
\text { of untreated tumors }\end{array}$ & $\begin{array}{l}\text { CCL2; CCL5; circular nuclei; T cell inactivation; } \\
\text { matrix degradation }\end{array}$ & Antiinflammatory subset with tumorigenic effects \\
\hline CD16 $6^{\text {dim }}$ CD62L bright & $\begin{array}{l}\text { Immature neutrophils identified in experimental } \\
\text { human models of endotoxemia }\end{array}$ & CD11 $^{10} ;$ CD11b $^{10} ;$ CD54 $^{10}$ & $\begin{array}{l}\text { Decreased ability to opsonize bacteria and } \\
\text { generate ROS }\end{array}$ \\
\hline IFN" ${ }^{\mathrm{hi}}$ & Found in healthy subjects & $\begin{array}{l}\text { Increased expression of IFN-stimulated genes } \\
\text { in RNA sequencing }\end{array}$ & $\begin{array}{l}\text { Unclear; higher ISG expression in women } \\
\text { than men }\end{array}$ \\
\hline
\end{tabular}

be better defined whether this heterogeneity is genetically driven and/or secondary to differences in microbiome composition, circadian rhythms, hormonal challenges, transmigration, senescence, and/or specific tissue microenvironments. Identifying distinct neutrophil subsets that play prominent roles in disease states is key in selectively targeting pathogenic granulocytes without hampering important homeostatic functions of the neutrophil compartment as a whole. In this regard, the identification of a distinct subset of proinflammatory neutrophils, called low-density granulocytes (LDGs) because of their tendency to fractionate with PBMCs during density separation of whole blood, has contributed to highlighting the putative role of neutrophil heterogeneity and dysregulated neutrophil death in autoimmune diseases $(85,86,91)$.

NETs display many putative proinflammatory properties that will be discussed in detail in the next section. In addition to their role in activation of the immune system, neutrophils and NETs contribute to resolution of inflammation by degrading chemokines and cytokines and activating serine proteases that decrease neutrophil recruitment (92). NETs can enhance Th2 cytokine expression, decrease Th1/Th17 cytokines (93), and induce both pro- and antiinflammatory effects on DCs (Figure 1 and refs. 30, 94). Neutrophils and NETs play a role in clearance of damaged endothelial cells and remodeling of senescent vessels in some tissues (95).

Role of neutrophils, LDGs, and NETs in SLE pathogenesis. A plethora of phenotypic and functional abnormalities have been described in lupus neutrophils (Figure 1), including increased cell death, impaired phagocytosis, and dysregulated oxidative activity $(19,47,55,85,86)$. In SLE bone marrow, neutrophils contribute to type I IFN synthesis and B cell dysregulation through BAFF-mediated effects (96).

Lupus LDGs are considered a distinct neutrophil subset with pathogenic roles. They differ from normal-density lupus and healthy control neutrophils at the transcriptomic, epigenetic, and functional levels $(19,85,86,90)$. LDGs are key drivers of the characteristic type I IFN gene signature of SLE and contribute to synthesis of type I IFNs and other proinflammatory cytokines. Lupus LDGs differ in protein content and have distinct modulation of their cytoskeleton compared with normal-density control and lupus neutrophils. This translates into differences in modulation of biomechanical properties that enhance their retention in microvasculature mimetics, suggesting that these cells may be more prone to become trapped in organs like the lung and induce tissue damage (90). LDGs are not a homogenous population and display transcriptional, epigenetic, and functional heterogeneity, comprising two main subpopulations of intermediate-mature and immature neutrophils, with various degrees of chromatin accessibility and distinct differences in transcription factor motif analysis (Table 2 and ref. 86). These two LDG subsets differ in various functional readouts, including phagocytosis, responses to type I IFN stimulation, NET formation, and chemotaxis. Overall, the bulk of SLE LDGs do not represent immature neutrophils, and most of them have enhanced proinflammatory roles. Furthermore, they do not display suppressive capabilities characteristic of myeloid-derived suppressor cells (97).

LDGs isolated from subjects with SLE (85) and other autoimmune and autoinflammatory conditions (54, 56, 83, 98-100) exhibit enhanced propensity to form NETs and damage endothelial cells, striated muscle cells, and other targets $(19,56)$. NETs also promote endothelial dysfunction by inducing vascular leakage and endothelial-mesenchymal transition through their ability to degrade vascular endothelial cadherin and activate $\beta$-catenin signaling, with implications for lupus nephritis (101). LDG NETs are more immunostimulatory and proinflammatory than NETs generated by other stimuli, with a higher abundance of modified 
self-antigens, including oxidized nucleic acids, and immunogenic/ proinflammatory molecules such as LL37 and MMP-9 $(19,55,102)$. NETs activate the NLRP3 inflammasome in macrophages, promoting enhanced release of IL-1 and IL-18, a process that is heightened in lupus macrophages and can perpetuate tissue damage (103).

Dysregulated NET formation plays a number of putative crucial pathogenic roles in SLE. In genetically predisposed individuals, key autoantigens that are externalized by NET formation may be presented to the immune system. Several genetic polymorphisms associated with SLE risk can enhance NET generation. For example, genetic alterations in TNFAIP3, encoding A20, disrupt the protein's deubiquitinase domain, promoting upregulation of protein citrullination and NET formation through increased PADI4 (104). Increased circulating NET complexes have been reported in SLE patients with a STAT4 risk allele $(105,106)$. Healthy individuals who are homozygous for the major SLE risk haplotype of IRF5 display increased spontaneous NET formation, supporting dysregulated neutrophil function as an early mechanism promoting autoimmunity (Table 1 and ref. 107).

Oxidation of nucleic acids and certain posttranslational modifications of proteins distinctly occur during NET formation and may promote enhanced immunogenicity. Both genomic and mitochondrial DNA become oxidized during NET formation. Enhanced nucleic acid oxidation enhances the interferogenic potential of NETs by extending the nucleic acids' half-life through resistance to degradation by nucleases, thereby effectively engaging intracellular DNA sensors that promote type I IFN responses, including the cGAS/STING pathway $(55,108)$. Ribonuclear protein ICs characteristic of SLE enhance mitochondrial ROS generation, which promotes oxidation of nucleic acids and NET formation (55). LDG NETs also display higher levels of oxidized nucleic acids than NETs purified from other sources, leading to higher induction of type I IFN responses in target cells (55). As such, the generation of modified nucleic acids by oxidation during pathogenic NET formation in SLE may be a key mechanism enhancing autoantigen halflife and induction of type I IFN and other inflammatory responses, promoting loss of tolerance. LDG NETs also display enhanced expression of ubiquitinated proteins that increase activation of proinflammatory responses in SLE macrophages (109). Overall, these observations indicate that not all NETs are generated equal, as lupus NETs are substantially more immunogenic and proinflammatory than NETs generated in other conditions.

The exact stimuli and pathways that lead to generation of NETs (59) may determine their role in lupus pathogenesis. For example, lupus-prone mice that lack NOX2, an enzyme involved in certain types of NET formation, develop exacerbated SLE (110), and patients with chronic granulomatous disease (CGD), characterized by impaired NOX activity, have a higher prevalence of autoimmunity. CGD patients also display an LDG subset with enhanced ability to form NETs with enhanced mitochondrial ROS synthesis and enhanced generation of IFN responses (55). In addition, genetic polymorphisms that impair NOX activity (NCF2) are associated with enhanced risk of developing SLE (111) and with accelerated lupus and enhanced NET activity in mouse models. As such, it is possible that a dysfunctional NOX pathway promotes synthesis of alternative sources of ROS (such as the mitochondria) that lead to aberrant pathways of nucleic acid oxidation, mitochon- drial dysfunction, and NET formation in SLE. This is supported by observations that inhibitors of mitochondrial dysfunction or mitochondrial ROS generation ameliorate murine lupus (55, 112-114). Similarly, the effectiveness of targeting PAD4 as a mechanism to decrease NET generation and lupus in animal models appears to be strain and model dependent, suggesting that models associated with more aberrant innate immune dysfunction improve with NET inhibition through PAD4 modulation (115).

NETs can directly modulate adaptive immune responses in SLE. LL37-DNA ICs activate endosomal TLRs to drive polyclonal B cell activation and expand self-reactive memory B cells (116). B cells can internalize and process NET components, and present them on class II MHC molecules to autoreactive T cells, promoting generation of memory $\mathrm{T}$ cells. NETs directly affect $\mathrm{T}$ cells by decreasing their activation threshold via Zap70-mediated phosphorylation of the $\mathrm{T}$ cell receptor, promoting enhanced $\mathrm{T}$ cell responses to suboptimal dosage of antigens (117). Importantly, the internalization of NETs through various ligand-receptor interactions can also drastically alter the function of other cells, including DCs and fibroblasts $(30,58,94)$.

In addition to enhanced capacity of LDGs to form NETs, a subset of SLE patients have impaired clearance of circulating NET components, which leads to increased half-life of modified self-antigens and their immunostimulatory effects and association with higher incidence of lupus nephritis (24). Various culprits for disruptions in NET clearance have been proposed, including complement activation within NETs, nucleic acid oxidation $(108,118$, 119), DNase I inhibitors, and anti-NET antibodies (24). Genetic polymorphisms that impact the function of molecules involved in removal of nucleic acids can impair NET clearance. For example, loss-of-function variants in DNASE1L3, encoding an extracellular nuclease endowed with the ability to degrade NETs, have been reported in familial forms of severe SLE (120).

Dysregulated NET formation/clearance appears to be operational in vivo, as neutrophils and NETs are increased in affected lupus skin, kidneys, and placenta, in association with tissue inflammation and organ damage $(19,121,122)$. At this point, it has not been possible to differentiate which neutrophil subsets infiltrate lupus tissues, as no distinct cell surface markers differentiate LDGs from other lupus neutrophils. Given recent advances in differentiating these cells at the transcriptional, epigenetic, and protein levels $(86,90)$, it may be possible in the future to expand these tools to identify the role of LDGs in vivo at the tissue level.

\section{LDGs and NETs in vascular damage in SLE}

Accumulating evidence implicates NETs in the development of vascular damage and atherothrombosis $(2,19,85,102,123,124)$. NETs induce endothelial cell apoptosis through an MMP-9/ MMP-2 axis that is enhanced in lupus LDG NETs (102). NETs harm vascular smooth muscle cells through a histone-dependent process that induces lytic cell death and promotes instability of atherosclerotic plaques (125). Various mouse models of atherosclerosis and vasculopathy suggest that inhibiting NET formation can alter susceptibility to plaque formation/inflammation and thrombosis $(124,126-128)$. Overall, dysregulated NET formation in the context of SLE may play prominent roles in the accelerated vascular damage characteristic of this disease. 
SLE subjects demonstrate vascular inflammation, arterial dysfunction, and increased noncalcified plaque burden, which may explain the higher reported risk for acute coronary syndromes (2, 123). Further supporting the role of LDGs in accelerated vascular damage in SLE, circulating numbers of these cells, as well as an LDG transcriptional signature, associate with increased coronary plaque and aortic wall inflammation, independent of other vascular risk factors $(86,123)$. Furthermore, NETs can oxidize HDL and disrupt its atheroprotective role, in part by modifying the ability of this lipoprotein to remove cholesterol from macrophages (cholesterol efflux capacity [CEC]) (129). HDL oxidation by NETs promotes proinflammatory responses in macrophages because the modified lipoprotein signals through the oxidized LDL receptor-1 (LOX-1), enhances NF- $\kappa$ B signaling, and disrupts the ability of HDL to block TLR-induced pathways (129). Overall, these observations suggest pleiotropic vasculopathic roles of lupus LDGs and NETs.

\section{Pathogenic crosstalk between IFNs and neutrophils in SLE}

IFN pathway dysregulation plays critical roles in lupus pathogenesis (130). A significant proportion of individuals diagnosed with SLE display an elevated IFN signature in peripheral blood and/or affected tissues. Altered regulation of this pathway is likely an early event, since genetic polymorphisms that impact type I IFN signaling associate with SLE risk (Table 1 and refs. 105, 131, 132). Genetically predisposed individuals may have a lower threshold to activate the type I IFN pathway when exposed to exogenous stimuli (viral, ultraviolet light) or endogenous stimuli (dead cells, ICs, NETs) $(55,133,134)$. In vitro and in vivo human and murine studies highlight the type I IFN pathway's importance in immune dysregulation and vascular and tissue damage $(106,135-139)$. Plasmacytoid DC activation is a main driver for enhanced production of IFNs, but other cell types, particularly of myeloid origin, also contribute $(55,85,140,141)$. IFNs enhance expression of costimulatory molecules and antigen presentation by antigen-presenting cells (142) and alter B cell tolerance, plasma cell differentiation, immunoglobulin class switching, antibody production, and generation of long-lasting memory B cells (143-147). Type I IFNs prolong T cell survival, inhibit T cell apoptosis, enhance activity of cytotoxic T cells, suppress Treg activity, and modify $\mathrm{T}$ cell polarization $(142,148,149)$.

In the context of neutrophil dysregulation, type I IFNs can prime neutrophils to form NETs $(47,86,150)$ and may enhance their response to TLR agonists (151). Conversely, as NETs induce IFN production, this may lead to a vicious cycle of persistent inflammation (Figure 1). Compared with other immune cell types, LDGs display increased expression of ISGs (86) and increased type I IFN-induced proteins (90). Healthy young adult females display a neutrophil-specific increased ISG expression compared with males, with associated hyperresponsiveness to type I IFNs (66). This enhanced priming of female neutrophils may lead to increased responses to various danger signals. The neutrophils that express enhanced ISGs represent a distinct circulating neutrophil subset detected in healthy individuals, suggesting a putative critical role in antimicrobial and proinflammatory responses (Table 2 and refs. 66, 152).

Synergizing with the effects of neutrophils, type I IFNs may play a key role in triggering vasculopathy and premature athero- sclerosis characteristic of SLE. Elevated levels of IFN-regulated proteins and ISGs associate with SLE vascular disease (119) independent of traditional Framingham risk factors (153). Type I IFNs impair endothelial function (154) and hamper vascular repair in SLE (155). In lupus and atherosclerosis mouse models, type I IFNs impair vasculogenesis and promote endothelial dysfunction, atherothrombosis, and plaque progression (137). IFNs inhibit endothelial NO synthase and impair NO production by endothelial cells (156). Additionally, type I IFNs promote diet-induced insulin resistance by triggering liver accumulation of $\mathrm{CD} 8^{+} \mathrm{T}$ cells with subsequent glucose dysregulation and hepatic inflammation (157). Type I IFNs stimulate macrophage recruitment to arteries (158), promote foam cell formation (159), and predict worse outcome during myocardial infarction (160). ISGs are upregulated in bone marrow neutrophil/monocyte progenitors and circulating and cardiac neutrophils soon after myocardial infarction (161). Blocking type I IFN signaling using an anti-IFNAR1 mAb (anifrolumab) decreases circulating NET complexes and improves CEC, implying that type I IFN inhibition may improve cardiometabolic risk in SLE patients (138).

Other IFNs - type II (IFN- $\gamma$ ) and type III (IFN- $\lambda 1$, IFN- $\lambda 2$, IFN- $\lambda 3$ ) - may contribute to SLE pathogenesis. Variations in circulating levels of type I, II, and III IFNs and tissue responses may help explain heterogeneity in pattern of organ involvement and IFN signature in SLE $(15,162,163)$. Exogenous administration of IFN- $\gamma$ can worsen human SLE (164), and elevations in IFN- $\gamma$ associate with disease activity. An IFN- $\gamma$ signaling pathway is activated in SLE PBMCs (165), and elevated IFN- $\gamma$ in SLE T cells promotes monocyte/macrophage induction of BAFF (166). IFN- $\gamma$ may affect lupus B cell function and germinal center responses $(167,168)$. Increases in type III IFNs have been reported in SLE, in correlation with disease activity $(169,170)$. In a lupus mouse model, type III IFNs were shown to have a pathogenic role that is nonredundant relative to type I IFNs by promoting systemic immune dysregulation and local inflammatory effects in skin and kidneys (171). B cells are the main immune responders to type III IFNs in humans, with potentially important implications in SLE that require further investigation (172).

Overall, IFNs play key roles in SLE pathogenesis, and the relevance of the various types of IFNs in explaining the heterogeneity of SLE continues to be elucidated and may be of great importance in the design of therapies that can target their deleterious effects (Table 3). A pathogenic crosstalk between IFNs and neutrophils may play fundamental roles in initiating, amplifying, and maintaining various aspects of lupus immune dysregulation, as well as in long-term outcomes driven by the development of vasculopathy and end-stage organ damage (Figure 1).

\section{Targeting neutrophils, NETs, and IFNs in SLE}

Given the growing evidence for a fundamental role of the pathogenic interplay between type I IFNs, neutrophils, and NETs in SLE, there is interest in targeting them for therapeutic purposes. Decreasing the formation, release, and/or half-life of NETs can be attempted by a variety of strategies (Table 3). Targeting of oxidative pathways implicated in NET formation using NOX (54, 172) or mitochondrial ROS (55) inhibitors, or ROS scavengers like $\mathrm{N}$-acetylcysteine (NAC) (173), has been explored in vitro and in 


\section{Table 3. Potential therapeutics that target the IFN/neutrophil pathogenic crosstalk in SLE}

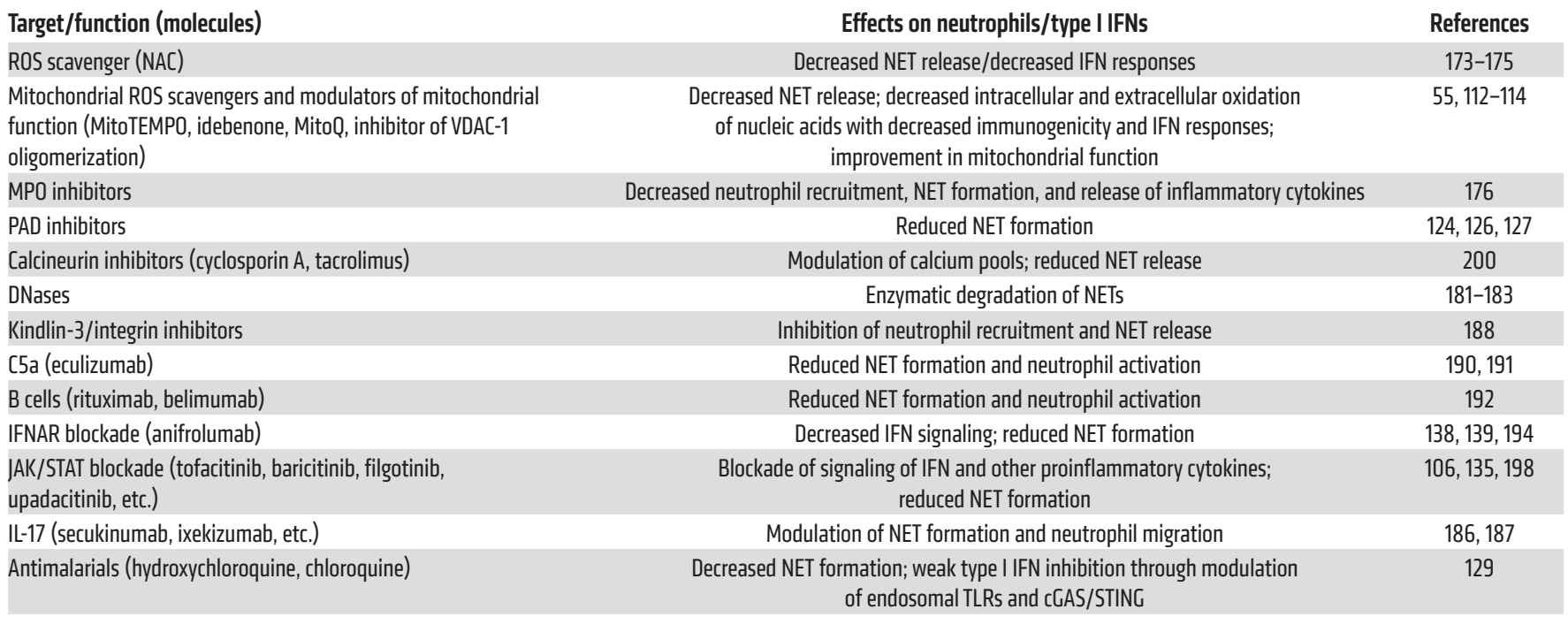

vivo. NAC decreased disease activity in SLE patients $(174,175)$, while inhibition of mitochondrial ROS or targeting of aberrant mitochondrial function hampered murine lupus severity $(55,112$, 113). In contrast, NOX inhibition may exacerbate SLE as a result of complex immunoregulatory effects (110). Inhibition of the function of various granule proteins can decrease neutrophil recruitment, NET formation, and inflammatory cytokine synthesis (176) and may be explored in future human studies. In preclinical studies, genetic deletion of PAD2 or PAD4 reduced ISGs, autoantibodies, vascular dysfunction, and clinical manifestations in TLR7dependent murine lupus, but not in other lupus models $(115,177)$. Inhibiting PAD enzymes using small molecules also decreases NET formation and protects from organ damage and vascular disease in various mouse models of lupus and vascular damage (124, $126,127)$. Given the heterogeneity of response in murine systems, future studies in humans will lead to further clarification of the role of PAD inhibition in SLE.

The putative deleterious effects on antimicrobial responses following NET inhibition in humans remain to be determined. PAD4-knockout mice maintain other antibacterial neutrophil functions even if NETs are inhibited. Even in mouse strains in which PAD inhibition does not totally abolish NET formation, the immunogenic and vasculopathic effects of these NETs are diminished, suggesting that partial enzymatic blockade may be sufficient $(177,178)$. Patients with genetic disorders that lead to deficiency of molecules implicated in NET formation (PADs, cathepsin C) are not overtly immunosuppressed $(179,180)$. However, additional preclinical and clinical studies are warranted to assess the safety profile, in different patient populations, of strategies that specifically inhibit NETs, without considerably altering other essential neutrophil functions.

Degradation of already formed NETs to enhance their remov$\mathrm{al}$ is another potential approach (181). Administration of DNase I to lupus-prone mice was beneficial (182), but early phase Ib studies of DNase I, though well tolerated, showed no changes in dis- ease markers, perhaps owing more to the half-life of the molecule used than to a lack of biological effects (183). DNaseIL3 is involved in degradation of extracellular DNA, like in NETs, and genetic deficiency of this enzyme in mice and humans is associated with autoantibody production and lupus features (184). Future studies could assess the role of this enzyme in improving NET clearance in SLE. Other drugs that can affect NET structures by destabilizing the neutrophil's actin cytoskeleton have also been proposed as potential therapies (185).

Targeting IL-17 signaling can modulate neutrophil trafficking and NET formation and may mitigate lupus glomerulonephritis $(186,187)$. Inhibition of neutrophil recruitment and NET release in tissues may also be accomplished by interruption of interactions between endothelial ligands and neutrophil-expressed kindlin-3 and $\beta_{2}$ integrin and other similar modalities (188). Targeting various chemokines may also limit pathogenic neutrophil infiltration in tissues (189). While inhibition of NET formation induced by C5a priming is an important pathway to consider, early clinical trials using anti-C5 antibodies, although well tolerated, did not promote improvements in disease activity even if they were effective in murine lupus $(190,191)$. In contrast, decreasing autoantibodies and ICs through a combination of mAbs targeting CD20 (rituximab) and BLyS/BAFF (belimumab) showed some benefits in lupus biomarkers and reduced circulating NETs (192), although the benefit of combined therapy versus single therapy remains to be better defined.

Targeting the IFN pathway holds promise for SLE management (Table 3). Blocking mAbs that target IFN- $\alpha$, IFN- $\gamma$, or IFNAR1 have been studied in SLE (193). Anifrolumab, which blocks IFNAR1, thus inhibiting the action of all type I IFNs, has shown promising results in one of two phase III trials, with a good tolerability profile and improvement in disease activity $(139,194)$. Anifrolumab decreased NET formation in SLE subjects when compared with placebo and improved the function of lupus HDL and other cardiometabolic parameters (138). These findings suggest 
that suppressing the IFN/neutrophil axis may help prevent longterm vascular complications in SLE and other diseases in which these pathways are dysregulated. Several medications currently used to treat SLE can modulate - at different levels - NET formation and/or the type I IFN signature. Antimalarials - drugs with proven benefit in SLE, including CVD benefits - can decrease NET formation in vitro (129) and are weak type I IFN inhibitors, potentially through modulation of endosomal TLRs (195) and the cGAS/STING pathway (196).

JAKs play fundamental roles in downstream signaling of multiple proinflammatory cytokines, including all IFNs (197). Additionally, JAKs directly modulate neutrophil biology. Tofacitinib, a pan-JAK inhibitor, reduced NET formation in vitro and in vivo and decreased disease manifestations, including vasculopathy, in lupus-prone mice (135). The JAK inhibitor baricitinib improved lupus clinical features in a phase II study (198), with phase III studies ongoing (NCT03616964, NCT03616912, ClinicalTrials. gov). A phase Ia/IIb clinical trial using tofacitinib in mild to moderate SLE showed significant decreases in type I IFN signature and circulating LDGs and NETs, with associated improvement in vascular function and cardiometabolic parameters (106). These observations support that targeting the neutrophil/IFN crosstalk with JAK/STAT inhibitors may modulate lupus vasculopathy and should be further explored in CVD prevention in SLE (106).

\section{Concluding remarks and remaining questions}

SLE is complex and heterogeneous, with clear dysfunction in most components of the immune system and therapeutic challenges given pathogenic and clinical heterogeneity. Dysregulation in crucial innate immune pathways implicated in host defense has profound implications in various aspects of lupus pathogenesis, including break of tolerance, induction of IFNs and other proinflammatory cytokines, aberrant adaptive immunity, and tissue damage. Accumulating in vitro and in vivo evidence, using human and murine systems, supports a central role for neutrophil dysregulation in SLE pathogenesis, spanning loss of tolerance, induction and amplification of inflammatory pathways, tissue damage, vascular disease, and cardiometabolic dysfunction. Future studies will hopefully help to better define subsets of patients in whom neutrophil/IFN interactions play particularly fundamental roles, in order to design better personalized therapeutic approaches. Further understanding the effects of the interaction between genetic, epigenetic, and various environmental stimuli on IFN responses, neutrophil heterogeneity, and the composition of NETs may help to explain the variability of SLE manifestations and the diverse autoantibody responses. Future studies should focus on better defining the mechanisms that regulate the cross-play between IFN and myeloid cells, and downstream consequences in autoimmunity, in order to identify promising therapies. This has implications for other chronic inflammatory diseases in which type I IFNs, neutrophils, and NETs are proposed to play important pathogenic roles.

Several important questions remain, and the exact role of these immune players in disease pathogenesis may only be understood if clinical trials that specifically target neutrophil dysregulation, aberrant NET formation, and the neutrophil/IFN crosstalk in SLE come to fruition. One important aspect is whether it will be feasible to specifically target LDGs, while leaving other neutrophil subsets untouched. This will depend on further advances in the identification of unique markers of this neutrophil subset and a better understanding of the genetic, epigenetic, and environmental factors that modulate granulocyte plasticity in health and disease states. While significant advances have occurred in characterizing LDGs, the factors that promote their formation, release, and pathogenicity (genetic or acquired) remain unclear. The extent to which NETs contribute to the various aspects of lupus pathogenesis and why only certain autoantibodies promote NET formation remain to be clarified. Understanding why different autoimmune diseases, characterized by enhanced NET formation, are associated with distinct autoantibody responses that target specific NET components will help better characterize heterogeneity of the NET protein and nucleic acid cargo and the role that genetics play in the adaptive immune response to these structures. This may lead to the identification of better molecular candidates as therapies to hopefully improve clinical outcomes.

\section{Acknowledgments}

This research was supported by the Intramural Research Program of the National Institute of Arthritis and Musculoskeletal and Skin Diseases of the NIH.

Address correspondence to: Mariana J. Kaplan, Systemic Autoimmunity Branch, NIAMS/NIH, 10 Center Drive, 12N248C, Bethesda, Maryland 20892, USA. Phone: 301.496.0517; Email: mariana. kaplan@nih.gov.
1. Tsokos GC. Systemic lupus erythematosus. N Engl JMed. 2011;365(22):2110-2121.

2. Liu Y, Kaplan MJ. Cardiovascular disease in systemic lupus erythematosus: an update. Curr Opin Rheumatol. 2018;30(5):441-448.

3. Tsokos GC, et al. New insights into the immunopathogenesis of systemic lupus erythematosus. Nat Rev Rheumatol. 2016;12(12):716-730.

4. Arbuckle MR, et al. Development of autoantibodies before the clinical onset of systemic lupus erythematosus. $N$ Engl J Med. 2003;349(16):1526-1533.

5 . Niewold TB, et al. High serum IFN-alpha activity is a heritable risk factor for systemic lupus erythematosus. Genes Immun. 2007;8(6):492-502.

6 . Herrada AA, et al. Innate immune cells' contri- bution to systemic lupus erythematosus. Front Immunol. 2019;10:772.

7. Durcan L, et al. Management strategies and future directions for systemic lupus erythematosus in adults. Lancet. 2019;393(10188):2332-2343.

8. Katsiari CG, et al. The pathophysiologic role of monocytes and macrophages in systemic lupus erythematosus: a reappraisal. Semin Arthritis Rheum. 2010;39(6):491-503.

9. Iwata Y, et al. Aberrant macrophages mediate defective kidney repair that triggers nephritis in lupus-susceptible mice. JImmunol. 2012;188(9):4568-4580.

10. Menon M, et al. A regulatory feedback between plasmacytoid dendritic cells and regulatory B cells is aberrant in systemic lupus erythematosus.
Immunity. 2016;44(3):683-697.

11. Ding D, et al. Aberrant phenotype and function of myeloid dendritic cells in systemic lupus erythematosus. J Immunol. 2006;177(9):5878-5889.

12. Charles N, et al. Basophils and the T helper 2 environment can promote the development of lupus nephritis. Nat Med. 2010;16(6):701-707.

13. Gupta S, Kaplan MJ. The role of neutrophils and NETosis in autoimmune and renal diseases. Nat Rev Nephrol. 2016;12(7):402-413.

14. Botto M, Walport MJ. C1q, autoimmunity and apoptosis. Immunobiology. 2002;205(4-5):395-406.

15. Oke V, et al. High levels of circulating interferons type I, type II and type III associate with distinct clinical features of active systemic lupus erythematosus. Arthritis Res Ther. 2019;21(1):107. 
16. Nicholls AJ, et al. Activation of the sympathetic nervous system modulates neutrophil function. J Leukoc Biol. 2018;103(2):295-309.

17. Elkon KB. Review: Cell death, nucleic acids, and immunity: inflammation beyond the grave. Arthritis Rheumatol.2018;70(6):805-816.

18. Mistry P, Kaplan MJ. Cell death in the pathogenesis of systemic lupus erythematosus and lupus nephritis. Clin Immunol. 2017;185:59-73.

19. Villanueva E, et al. Netting neutrophils induce endothelial damage, infiltrate tissues, and expose immunostimulatory molecules in systemic lupus erythematosus. JImmunol. 2011;187(1):538-552.

20. Kahlenberg JM, et al. An essential role of caspase 1 in the induction of murine lupus and its associated vascular damage. Arthritis Rheumatol. 2014;66(1):152-162.

21. Kahlenberg JM, et al. Inflammasome activation of IL-18 results in endothelial progenitor cell dysfunction in systemic lupus erythematosus. JImmunol. 2011;187(11):6143-6156.

22. Denny MF, et al. Accelerated macrophage apoptosis induces autoantibody formation and organ damage in systemic lupus erythematosus. J Immunol.2006;176(4):2095-2104.

23. Munoz LE, et al. The role of defective clearance of apoptotic cells in systemic autoimmunity. Nat Rev Rheumatol. 2010;6(5):280-289.

24. Hakkim A, et al. Impairment of neutrophil extracellular trap degradation is associated with lupus nephritis. Proc Natl Acad Sci U S A. 2010;107(21):9813-9818.

25. Dieker J, et al. Circulating apoptotic microparticles in systemic lupus erythematosus patients drive the activation of dendritic cell subsets and prime neutrophils for NETosis. Arthritis Rheumatol. 2016;68(2):462-472.

26. Watanabe-Fukunaga R, et al. Lymphoproliferation disorder in mice explained by defects in Fas antigen that mediates apoptosis. Nature. 1992;356(6367):314-317.

27. Wilber A, et al. Dnase113 deficiency in lupusprone MRL and NZB/W F1 mice. Clin Exp Immunol. 2003;134(1):46-52.

28. Strasser A, et al. Enforced BCL2 expression in B-lymphoid cells prolongs antibody responses and elicits autoimmune disease. Proc Natl Acad Sci U S A. 1991;88(19):8661-8665.

29. Baumann I, et al. Impaired uptake of apoptotic cells into tingible body macrophages in germinal centers of patients with systemic lupus erythematosus. Arthritis Rheum. 2002;46(1):191-201.

30. Lande R, et al. Neutrophils activate plasmacytoid dendritic cells by releasing self-DNA-peptide complexes in systemic lupus erythematosus. Sci Transl Med. 2011;3(73):73ra19.

31. Leonard $\mathrm{D}$, et al. Activated $\mathrm{T}$ cells enhance interferon- $\alpha$ production by plasmacytoid dendritic cells stimulated with RNA-containing immune complexes. Ann Rheum Dis. 2016;75(9):1728-1734.

32. Nauseef WM, Borregaard N. Neutrophils at work. Nat Immunol. 2014;15(7):602-611.

33. Ballesteros I, et al. Co-option of neutrophil fates by tissue environments. Cell. 2020;183(5):1282-1297.e18.

34. Aroca-Crevillen A, et al. Circadian features of neutrophil biology. Front Immunol. 2020;11:576.
35. Noubouossie DF, et al. Neutrophils: back in the thrombosis spotlight. Blood. 2019;133(20):2186-2197.

36. Fuchs TA, et al. Extracellular DNA traps promote thrombosis. Proc Natl Acad Sci U S A. 2010;107(36):15880-15885.

37. Massena S, et al. Identification and characterization of VEGF-A-responsive neutrophils expressing CD49d, VEGFR1, and CXCR4 in mice and humans. Blood. 2015;126(17):2016-2026.

38. Wang J, et al. Visualizing the function and fate of neutrophils in sterile injury and repair. Science. 2017;358(6359):111-116.

39. Christoffersson G, Phillipson M. The neutrophil: one cell on many missions or many cells with different agendas? Cell Tissue Res. 2018;371(3):415-423.

40. Herter J, Zarbock A. Integrin regulation during leukocyte recruitment. J Immunol. 2013;190(9):4451-4457.

41. Henson PM. Pathologic mechanisms in neutrophil-mediated injury. Am J Pathol. 1972;68(3):593-612.

42. Ley K, et al. Neutrophils: new insights and open questions. Sci Immunol. 2018;3(30):eaat4579.

43. Brinkmann V, et al. Neutrophil extracellular traps kill bacteria. Science. 2004;303(5663):1532-1535.

44. Rorvig S, et al. Proteome profiling of human neutrophil granule subsets, secretory vesicles, and cell membrane: correlation with transcriptome profiling of neutrophil precursors. J Leukoc Biol. 2013;94(4):711-721.

45. Yipp BG, et al. Infection-induced NETosis is a dynamic process involving neutrophil multitasking in vivo. Nat Med. 2012;18(9):1386-1393.

46. Yousefi S, et al. Viable neutrophils release mitochondrial DNA to form neutrophil extracellular traps. Cell Death Differ. 2009;16(11):1438-1444.

47. Garcia-Romo GS, et al. Netting neutrophils are major inducers of type I IFN production in pediatric systemic lupus erythematosus. Sci Transl Med.2011;3(73):73ra20.

48. Semeraro F, et al. Extracellular histones promote thrombin generation through platelet-dependen mechanisms: involvement of platelet TLR2 and TLR4. Blood. 2011;118(7):1952-1961.

49. Clark SR, et al. Platelet TLR4 activates neutrophil extracellular traps to ensnare bacteria in septic blood. Nat Med. 2007;13(4):463-469.

50. Carestia A, et al. Mediators and molecular pathways involved in the regulation of neutrophil extracellular trap formation mediated by activated platelets. J Leukoc Biol. 2016;99(1):153-162.

51. Keshari RS, et al. Cytokines induced neutrophil extracellular traps formation: implication for the inflammatory disease condition. PLoS One. 2012;7(10): 48111.

52. Schorn C, et al. Monosodium urate crystals induce extracellular DNA traps in neutrophils, eosinophils, and basophils but not in mononuclear cells. Front Immunol. 2012;3:277.

53. Munoz LE, et al. Neutrophil extracellular traps initiate gallstone formation. Immunity. 2019;51(3):443-450.e4.

54. Yalavarthi S, et al. Antiphospholipid antibodies promote the release of neutrophil extracellular traps: a new mechanism of thrombosis in the antiphospholipid syndrome. Arthritis Rheumatol.
2015;67(11):2990-3003.

55. Lood C, et al. Neutrophil extracellular traps enriched in oxidized mitochondrial DNA are interferogenic and contribute to lupus-like disease. Nat Med. 2016;22(2):146-153.

56 . Seto N, et al. Neutrophil dysregulation is pathogenic in idiopathic inflammatory myopathies. JCI Insight. 2020;5(3):e134189.

57. Behnen M, et al. Immobilized immune complexes induce neutrophil extracellular trap release by human neutrophil granulocytes via Fc $\gamma$ RIIIB and Mac-1. J Immunol. 2014;193(4):1954-1965.

58. Khandpur R, et al. NETs are a source of citrullinated autoantigens and stimulate inflammatory responses in rheumatoid arthritis. Sci Transl Med. 2013;5(178):178ra40

59. Kenny EF, et al. Diverse stimuli engage different neutrophil extracellular trap pathways. Elife. 2017;6:e24437.

60. Wang Y, et al. Histone hypercitrullination mediates chromatin decondensation and neutrophil extracellular trap formation. J Cell Biol. 2009;184(2):205-213.

61. Tessarz P, Kouzarides T. Histone core modifications regulating nucleosome structure and dynamics. Nat Rev Mol Cell Biol. 2014;15(11):703-708

62. Sollberger G, et al. Gasdermin D plays a vital role in the generation of neutrophil extracellular traps. Sci Immunol. 2018;3(26):eaar6689.

63. Chen KW, et al. Noncanonical inflammasome signaling elicits gasdermin D-dependent neutrophil extracellular traps. Sci Immunol. 2018;3(26):eaar6676

64. Wang X, et al. Effects of gasdermin D in modulating murine lupus and its associated organ damage. Arthritis Rheumatol. 2020;72(12):2118-2129.

65. Blazkova J, et al. Multicenter systems analysis of human blood reveals immature neutrophils in males and during pregnancy. Jimmunol. 2017;198(6):2479-2488.

66. Gupta S, et al. Sex differences in neutrophil biology modulate response to type I interferons and immunometabolism. Proc Natl Acad Sci U S A. 2020;117(28):16481-16491.

67. Megiovanni AM, et al. Polymorphonuclear neutrophils deliver activation signals and antigenic molecules to dendritic cells: a new link between leukocytes upstream of T lymphocytes. J Leukoc Biol. 2006;79(5):977-988.

68. Yang CW, et al. Neutrophils influence the level of antigen presentation during the immune response to protein antigens in adjuvants. J Immunol. 2010;185(5):2927-2934.

69. Schonrich G, et al. Devilishly radical NETwork in COVID-19: oxidative stress, neutrophil extracellular traps (NETs), and T cell suppression. Adv Biol Regul. 2020;77:100741.

70. Fazio J, et al. Inhibition of human $\gamma \delta \mathrm{T}$ cell proliferation and effector functions by neutrophil serine proteases. Scand J Immunol. 2014;80(6):381-389.

71. Rodriguez PC, et al. Arginase I production in the tumor microenvironment by mature myeloid cells inhibits T-cell receptor expression and antigen-specific T-cell responses. Cancer Res. 2004;64(16):5839-5849.

72. Bowers NL, et al. Immune suppression by neutrophils in HIV-1 infection: role of PD-L1/PD-1 
pathway. PLoS Pathog. 2014;10(3):e1003993.

73. Curran CS, et al. PD-1 immunobiology in systemic lupus erythematosus. JAutoimmun. 2019;97:1-9.

74. Tvinnereim AR, et al. Neutrophil involvement in cross-priming $\mathrm{CD} 8^{+} \mathrm{T}$ cell responses to bacterial antigens. J Immunol. 2004;173(3):1994-2002.

75. Lim K, et al. In situ neutrophil efferocytosis shapes $\mathrm{T}$ cell immunity to influenza infection. Nat Immunol. 2020;21(9):1046-1057.

76. Puga I, et al. B cell-helper neutrophils stimulate the diversification and production of immunoglobulin in the marginal zone of the spleen. Nat Immunol. 2012;13(2):170-180.

77. Parsa R, et al. BAFF-secreting neutrophils drive plasma cell responses during emergency granulopoiesis. J Exp Med. 2016;213(8):1537-1553.

78. Giordano D, et al. BAFF produced by neutrophils and dendritic cells is regulated differently and has distinct roles in antibody responses and protective immunity against west nile virus. J Immunol. 2020;204(6):1508-1520.

79. Huard B, et al. APRIL secreted by neutrophils binds to heparan sulfate proteoglycans to create plasma cell niches in human mucosa. JClin Invest. 2008;118(8):2887-2895.

80. Manfroi B, et al. CXCL-8/IL8 produced by diffuse large B-cell lymphomas recruits neutrophils expressing a proliferation-inducing ligand APRIL. Cancer Res. 2017;77(5):1097-1107.

81. Chiu IM, et al. Bacteria activate sensory neurons that modulate pain and inflammation. Nature. 2013;501(7465):52-57.

82. Barletta KE, et al. Regulation of neutrophil function by adenosine. Arterioscler Thromb Vasc Biol. 2012;32(4):856-864.

83. Carmona-Rivera C, et al. Deficiency of adenosine deaminase 2 triggers adenosine-mediated NETosis and TNF production in patients with DADA2. Blood. 2019;134(4):395-406.

84. Ali RA, et al. Adenosine receptor agonism protects against NETosis and thrombosis in antiphospholipid syndrome. Nat Commun. 2019;10(1):1916.

85. Denny MF, et al. A distinct subset of proinflammatory neutrophils isolated from patients with systemic lupus erythematosus induces vascular damage and synthesizes type I IFNs. J Immunol. 2010;184(6):3284-3297.

86. Mistry P, et al. Transcriptomic, epigenetic, and functional analyses implicate neutrophil diversity in the pathogenesis of systemic lupus erythematosus. Proc Natl Acad Sci U S A. 2019;116(50):25222-25228.

87. Fridlender ZG, et al. Polarization of tumor-associated neutrophil phenotype by TGF-beta: "N1" versus "N2" TAN. Cancer Cell. 2009;16(3):183-194.

88. Ma Y, et al. Temporal neutrophil polarization following myocardial infarction. Cardiovasc Res. 2016;110(1):51-61.

89. Sagiv JY, et al. Phenotypic diversity and plasticity in circulating neutrophil subpopulations in cancer. Cell Rep. 2015;10(4):562-573.

90. Bashant KR, et al. Proteomic, biomechanical and functional analyses define neutrophil heterogeneity in systemic lupus erythematosus [published online September 28, 2020]. Ann Rheum Dis. https://doi.org/10.1136/ annrheumdis-2020-218338.
91. Hacbarth E, Kajdacsy-Balla A. Low density neutrophils in patients with systemic lupus erythematosus, rheumatoid arthritis, and acute rheumatic fever. Arthritis Rheum. 1986;29(11):1334-1342.

92. Schauer C, et al. Aggregated neutrophil extracellular traps limit inflammation by degrading cytokines and chemokines. Nat Med.2014;20(5):511-517.

93. Barrientos L, et al. Neutrophil extracellular traps downregulate lipopolysaccharide-induced activation of monocyte-derived dendritic cells. JImmunol. 2014;193(11):5689-5698.

94. Sangaletti S, et al. Neutrophil extracellular traps mediate transfer of cytoplasmic neutrophil antigens to myeloid dendritic cells toward ANCA induction and associated autoimmunity. Blood. 2012;120(15):3007-3018.

95. Binet F, et al. Neutrophil extracellular traps target senescent vasculature for tissue remodeling in retinopathy. Science. 2020;369(6506):eaay5356.

96. Palanichamy A, et al. Neutrophil-mediated IFN activation in the bone marrow alters $\mathrm{B}$ cell development in human and murine systemic lupus erythematosus. J Immunol. 2014;192(3):906-918

97. Rahman S, et al. Low-density granulocytes activate $\mathrm{T}$ cells and demonstrate a non-suppressive role in systemic lupus erythematosus. Ann Rheum Dis. 2019;78(7):957-966.

98. Kessenbrock K, et al. Netting neutrophils in autoimmune small-vessel vasculitis. Nat Med. 2009;15(6):623-625.

99. Lin AM, et al. Mast cells and neutrophils release IL-17 through extracellular trap formation in psoriasis. J Immunol. 2011;187(1):490-500.

100.Mistry P, et al. Dysregulated neutrophil responses and neutrophil extracellular trap formation and degradation in PAPA syndrome. Ann Rheum Dis. 2018;77(12):1825-1833.

101. Pieterse E, et al. Neutrophil extracellular traps drive endothelial-to-mesenchymal transition. Arterioscler Thromb Vasc Biol. 2017;37(7):1371-1379.

102. Carmona-Rivera C, et al. Neutrophil extracellular traps induce endothelial dysfunction in systemic lupus erythematosus through the activation of matrix metalloproteinase-2. Ann Rheum Dis. 2015;74(7):1417-1424

103. Kahlenberg JM, et al. Neutrophil extracellular trap-associated protein activation of the NLRP3 inflammasome is enhanced in lupus macrophages. JImmunol. 2013;190(3):1217-1226.

104. Odqvist L, et al. Genetic variations in A20 DUB domain provide a genetic link to citrullination and neutrophil extracellular traps in systemic lupus erythematosus. Ann Rheum Dis. 2019;78(10):1363-1370.

105. Remmers EF, et al. STAT4 and the risk of rheumatoid arthritis and systemic lupus erythematosus. N EnglJ Med. 2007;357(10):977-986.

106.Hasni S, et al. A phase 1b/2a trial of tofacitinib, an oral janus kinase inhibitor, in systemic lupus erythematosus. Lupus Sci Med. 2019;6(suppl 1):A139-A140.

107. Li D, et al. IRF5 genetic risk variants drive myeloid-specific IRF5 hyperactivation and presymptomatic SLE. JCI Insight. 2020;5(2):124020.

108. Gehrke N, et al. Oxidative damage of DNA confers resistance to cytosolic nuclease TREX1 degradation and potentiates STING-dependent immune sensing. Immunity. 2013;39(3):482-495.

109. Barrera-Vargas A, et al. Differential ubiquitination in NETs regulates macrophage responses in systemic lupus erythematosus. Ann Rheum Dis. 2018;77(6):944-950.

110.Campbell AM, et al. NADPH oxidase inhibits the pathogenesis of systemic lupus erythematosus. Sci Transl Med. 2012;4(157):157ra41.

111. Jacob CO, et al. Lupus-associated causal mutation in neutrophil cytosolic factor 2 (NCF2) brings unique insights to the structure and function of NADPH oxidase. Proc Natl Acad Sci U S A. 2012;109(2):E59-E67.

112. Blanco LP, et al. Improved mitochondrial metabolism and reduced inflammation following attenuation of murine lupus with coenzyme Q10 analog idebenone. Arthritis Rheumatol. 2020;72(3):454-464.

113. Fortner KA, et al. Targeting mitochondrial oxidative stress with MitoQ reduces NET formation and kidney disease in lupus-prone MRL-lpr mice. Lupus Sci Med. 2020;7(1):e000387.

114. Kim J, et al. VDAC oligomers form mitochondrial pores to release mtDNA fragments and promote lupus-like disease. Science. 2019;366(6472):1531-1536.

115. Gordon RA, et al. Lupus and proliferative nephritis are PAD4 independent in murine models. JCI Insight. 2017;2(10):e92926.

116. Gestermann N, et al. Netting neutrophils activate autoreactive B cells in lupus. J Immunol. 2018;200(10):3364-3371.

117. Tillack K, et al. $\mathrm{T}$ lymphocyte priming by neutrophil extracellular traps links innate and adaptive immune responses. JImmunol. 2012;188(7):3150-3159

118. Leffler J, et al. Neutrophil extracellular traps that are not degraded in systemic lupus erythematosus activate complement exacerbating the disease. JImmunol. 2012;188(7):3522-3531.

119. Lood C, et al. Platelet transcriptional profile and protein expression in patients with systemic lupus erythematosus: up-regulation of the type I interferon system is strongly associated with vascular disease. Blood. 2010;116(11):1951-1957.

120. Al-Mayouf SM, et al. Loss-of-function variant in DNASE1L3 causes a familial form of systemic lupus erythematosus. Nat Genet. 2011;43(12):1186-1188.

121. Marder W, et al. Placental histology and neutrophil extracellular traps in lupus and pre-eclampsia pregnancies. Lupus Sci Med. 2016;3(1):e000134.

122.Frangou E, et al. REDD1/autophagy pathway promotes thromboinflammation and fibrosis in human systemic lupus erythematosus (SLE) through NETs decorated with tissue factor (TF) and interleukin-17A (IL-17A). Ann Rheum Dis. 2019;78(2):238-248.

123. Carlucci PM, et al. Neutrophil subsets and their gene signature associate with vascular inflammation and coronary atherosclerosis in lupus. JCI Insight. 2018;3(8):e99276.

124. Knight JS, et al. Peptidylarginine deiminase inhibition disrupts NET formation and protects against kidney, skin and vascular disease in lupus-prone MRL/lpr mice. Ann Rheum Dis. 2015;74(12):2199-2206. 
125. Silvestre-Roig C, et al. Externalized histone H4 orchestrates chronic inflammation by inducing lytic cell death. Nature. 2019;569(7755):236-240.

126. Knight JS, et al. Peptidylarginine deiminase inhibition is immunomodulatory and vasculoprotective in murine lupus. JClin Invest. 2013;123(7):2981-2993.

127. Knight JS, et al. Peptidylarginine deiminase inhibition reduces vascular damage and modulates innate immune responses in murine models of atherosclerosis. Circ Res. 2014;114(6):947-956.

128. Liu Y, et al. Myeloid-specific deletion of peptidylarginine deiminase 4 mitigates atherosclerosis. Front Immunol. 2018;9:1680.

129. Smith CK, et al. Neutrophil extracellular trapderived enzymes oxidize high-density lipoprotein: an additional proatherogenic mechanism in systemic lupus erythematosus. Arthritis Rheumatol. 2014;66(9):2532-2544.

130. Crow MK. Type I interferon in the pathogenesis of lupus. J Immunol. 2014;192(12):5459-5468.

131. Langefeld CD, et al. Transancestral mapping and genetic load in systemic lupus erythematosus. Nat Commun. 2017;8:16021.

132. International Consortium for Systemic Lupus Erythematosus Genetics, et al. Genome-wide association scan in women with systemic lupus erythematosus identifies susceptibility variant in ITGAM, PXK, KIAA1542 and other loci. Nat Genet. 2008;40(2):204-210.

133. Mavragani CP, et al. Expression of long interspersed nuclear element 1 retroelements and induction of type I interferon in patients with systemic autoimmune disease. Arthritis Rheumatol. 2016;68(11):2686-2696.

134. Robinson T, et al. Autoimmune disease risk variant of IFIH1 is associated with increased sensitivity to IFN- $\alpha$ and serologic autoimmunity in lupus patients. JImmunol. 2011;187(3):1298-1303.

135. Furumoto Y, et al. Tofacitinib ameliorates murine lupus and its associated vascular dysfunction. Arthritis Rheumatol. 2017;69(1):148-160.

136. Moore E, Putterman C. Are lupus animal models useful for understanding and developing new therapies for human SLE? J Autoimmun. 2020;112:102490.

137. Thacker SG, et al. Type I interferons modulate vascular function, repair, thrombosis, and plaque progression in murine models of lupus and atherosclerosis. Arthritis Rheum. 2012;64(9):2975-2985.

138. Casey KA, et al. Modulation of cardiometabolic disease markers by type I interferon inhibition in systemic lupus erythematosus [published online September 10, 2020]. Arthritis Rheumatol. https://doi.org/10.1002/art.41518.

139. Morand EF, et al. Trial of anifrolumab in active systemic lupus erythematosus. $N$ Engl J Med. 2020;382(3):211-221.

140. Murayama G, et al. Inhibition of mTOR suppresses IFNalpha productionthe STING pathway in monocytes from systemic lupus erythematosus patients. Rheumatology (Oxford). 2020;59(10):2992-3002.

141. Buechler MB, et al. Cutting edge: Type I IFN drives emergency myelopoiesis and peripheral myeloid expansion during chronic TLR7 signaling. J Immunol. 2013;190(3):886-891.

142.Gonzalez-Navajas JM, et al. Immunomodulatory functions of type I interferons. Nat Rev Immunol. 2012;12(2):125-135.

143. Care MA, et al. Network analysis identifies proinflammatory plasma cell polarization for secretion of ISG15 in human autoimmunity. J Immunol. 2016;197(4):1447-1459.

144. Vazquez MI, et al. B cells responses and cytokine production are regulated by their immune microenvironment. Cytokine. 2015;74(2):318-326.

145. Kiefer K, et al. Role of type I interferons in the activation of autoreactive B cells. Immunol Cel Biol. 2012;90(5):498-504.

146. Giltiay NV, et al. The plasticity of newly formed B cells. J Immunol. 2019;203(12):3095-3104.

147. Domeier PP, et al. B-cell-intrinsic type 1 interferon signaling is crucial for loss of tolerance and the development of autoreactive B cells. Cell Rep. 2018;24(2):406-418.

148. Lopez P, et al. A pathogenic IFN $\alpha$, BLyS and IL-17 axis in systemic lupus erythematosus patients. $\mathrm{Sci}$ Rep. 2016;6:20651.

149. Alunno A, et al. Balance between regulatory $\mathrm{T}$ and Th17 cells in systemic lupus erythematosus: the old and the new. Clin Dev Immunol. 2012;2012:823085.

150. Gul E, et al. Type I IFN-related NETosis in ataxia telangiectasia and Artemis deficiency. JAllergy Clin Immunol. 2018;142(1):246-257.

151. Zimmermann M, et al. IFN $\alpha$ enhances the production of IL- 6 by human neutrophils activated via TLR8. Sci Rep. 2016;6:19674.

152. Xie X, et al. Single-cell transcriptome profiling reveals neutrophil heterogeneity in homeostasis and infection. Nat Immunol. 2020;21(9):1119-1133.

153. Somers EC, et al. Type I interferons are associated with subclinical markers of cardiovascular disease in a cohort of systemic lupus erythematosus patients. PLoS One. 2012;7(5):e37000.

154. Tyden H, et al. Endothelial dysfunction is associated with activation of the type I interferon system and platelets in patients with systemic lupus erythematosus. RMD Open. 2017;3(2):e000508.

155. Denny MF, et al. Interferon-alpha promotes abnormal vasculogenesis in lupus: a potential pathway for premature atherosclerosis. Blood. 2007;110(8):2907-2915

156 . Buie JJ, et al. IFN- $\alpha$ negatively regulates the expression of endothelial nitric oxide synthase and nitric oxide production: implications for systemic lupus erythematosus. JImmunol. 2017;199(6):1979-1988.

157. Ghazarian M, et al. Type I interferon responses drive intrahepatic $\mathrm{T}$ cells to promote metabolic syndrome. Sci Immunol. 2017;2(10):eaai7616.

158. Goossens P, et al. Myeloid type I interferon signaling promotes atherosclerosis by stimulating macrophage recruitment to lesions. Cell Metab. 2010;12(2):142-153.

159. Li J, et al. Interferon- $\alpha$ priming promotes lipid uptake and macrophage-derived foam cell formation: a novel link between interferon- $\alpha$ and atherosclerosis in lupus. Arthritis Rheum. 2011;63(2):492-502.

160. King KR, et al. IRF3 and type I interferons fuel a fatal response to myocardial infarction. Nat Med. 2017;23(12):1481-1487.

161. Calcagno DM, et al. The myeloid type I interferon response to myocardial infarction begins in bone marrow and is regulated by Nrf2-activated macrophages. Sci Immunol. 2020;5(51):eaaz1974.

162.Balomenos D, et al. Interferon-gamma is required for lupus-like disease and lymphoaccumulation in MRL-lpr mice. J Clin Invest. 1998;101(2):364-371.

163. Seery JP, et al. Antinuclear autoantibodies and lupus nephritis in transgenic mice expressing interferon gamma in the epidermis. J Exp Med. 1997;186(9):1451-1459.

164. Machold KP, Smolen JS. Interferon-gamma induced exacerbation of systemic lupus erythematosus. J Rheumatol. 1990;17(6):831-832.

165. Karonitsch T, et al. Activation of the interferon-gamma signaling pathway in systemic lupus erythematosus peripheral blood mononuclear cells. Arthritis Rheum. 2009;60(5):1463-1471.

166. Harigai M, et al. Excessive production of IFN-gamma in patients with systemic lupus erythematosus and its contribution to induction of B lymphocyte stimulator/B cell-activating factor/TNF ligand superfamily-13B. J Immunol. 2008;181(3):2211-2219.

167. Chodisetti SB, et al. Type II but not type I IFN signaling is indispensable for TLR7-promoted development of autoreactive B cells and systemic autoimmunity. JImmunol. 2020;204(4):796-809.

168. Munroe ME, et al. Altered type II interferon precedes autoantibody accrual and elevated type I interferon activity prior to systemic lupus erythematosus classification. Ann Rheum Dis. 2016;75(11):2014-2021.

169.Wu Q, et al. Interferon-lambda1 induces peripheral blood mononuclear cell-derived chemokines secretion in patients with systemic lupus erythematosus: its correlation with disease activity. Arthritis Res Ther. 2011;13(3):R88

170.Zickert A, et al. Interferon (IFN)- $\lambda$ is a potential mediator in lupus nephritis. Lupus Sci Med. 2016;3(1):e000170.

171. Goel RR, et al. Interferon lambda promotes immune dysregulation and tissue inflammation in TLR7-induced lupus. Proc Natl Acad Sci U S A 2020;117(10):5409-5419.

172. Remijsen Q, et al. Neutrophil extracellular trap cell death requires both autophagy and superoxide generation. Cell Res. 2011;21(2):290-304.

173. Patel S, et al. Nitric oxide donors release extracellular traps from human neutrophils by augmenting free radical generation. Nitric Oxide. 2010;22(3):226-234.

174. Garcia RJ, et al. Attention deficit and hyperactivity disorder scores are elevated and respond to $\mathrm{N}$-acetylcysteine treatment in patients with systemic lupus erythematosus. Arthritis Rheum. 2013;65(5):1313-1318.

175. Lai ZW, et al. N-acetylcysteine reduces disease activity by blocking mammalian target of rapamycin in T cells from systemic lupus erythematosus patients: a randomized, double-blind, placebo-controlled trial. Arthritis Rheum. 2012;64(9):2937-2946.

176. Zheng W, et al. PF-1355, a mechanism-based myeloperoxidase inhibitor, prevents immune complex vasculitis and anti-glomerular basement membrane glomerulonephritis. J Pharmacol Exp Ther. 2015;353(2):288-298.

177. Liu Y, et al. Peptidylarginine deiminases 2 and 4 modulate innate and adaptive immune 
responses in TLR-7-dependent lupus. JCI Insight. 2018;3(23):e124729.

178. Martinod K, et al. PAD4-deficiency does not affect bacteremia in polymicrobial sepsis and ameliorates endotoxemic shock. Blood. 2015;125(12):1948-1956.

179. Sulem P, et al. Identification of a large set of rare complete human knockouts. Nat Genet. 2015;47(5):448-452.

180. Sorensen OE, et al. Papillon-Lefèvre syndrome patient reveals species-dependent requirements for neutrophil defenses. J Clin Invest. 2014;124(10):4539-4548.

181. Jimenez-Alcazar M, et al. Host DNases prevent vascular occlusion by neutrophil extracellular traps. Science. 2017;358(6367):1202-1206.

182. Macanovic M, et al. The treatment of systemic lupus erythematosus (SLE) in NZB/W F1 hybrid mice; studies with recombinant murine DNase and with dexamethasone. Clin Exp Immunol. 1996;106(2):243-252.

183. Davis JC Jr, et al. Recombinant human Dnase I (rhDNase) in patients with lupus nephritis. Lupus. 1999;8(1):68-76.

184.Sisirak V, et al. Digestion of chromatin in apoptotic cell microparticles prevents autoimmunity. Cell. 2016;166(1):88-101.

185. Neeli I, et al. Regulation of extracellular chro- matin release from neutrophils. J Innate Immun. 2009;1(3):194-201.

186. Pisitkun P, et al. Interleukin-17 cytokines are critical in development of fatal lupus glomerulonephritis. Immunity. 2012;37(6):1104-1115.

187. Satoh Y, et al. A case of refractory lupus nephritis complicated by psoriasis vulgaris that was controlled with secukinumab. Lupus. 2018;27(7):1202-1206.

188. Xu Z, et al. Interaction of kindlin-3 and $\beta 2$-integrins differentially regulates neutrophil recruitment and NET release in mice. Blood. 2015;126(3):373-377.

189. Disteldorf EM, et al. CXCL5 drives neutrophil recruitment in TH17-mediated GN. JAm Soc Nephrol. 2015;26(1):55-66.

190.Wang Y, et al. Amelioration of lupus-like autoimmune disease in NZB/WF1 mice after treatment with a blocking monoclonal antibody specific for complement component C5. Proc Natl Acad Sci US A. 1996;93(16):8563-8568.

191. Rother RP, et al. Inhibition of terminal complement: a novel therapeutic approach for the treatment of systemic lupus erythematosus. Lupus. 2004;13(5):328-334.

192.Kraaij T, et al. The NET-effect of combining rituximab with belimumab in severe systemic lupus erythematosus. J Autoimmun. 2018;91:45-54.

193. Chasset F, Arnaud L. Targeting interferons and their pathways in systemic lupus erythematosus. Autoimmun Rev. 2018;17(1):44-52.

194.Furie RA, et al. Type I interferon inhibitor anifrolumab in active systemic lupus erythematosus (TULIP-1): a randomised, controlled, phase 3 trial. Lancet Rheumatology. 2019;1(4):e208-e219.

195. Sacre K, et al. Hydroxychloroquine is associated with impaired interferon-alpha and tumor necrosis factor-alpha production by plasmacytoid dendritic cells in systemic lupus erythematosus. Arthritis Res Ther. 2012;14(3):R155.

196. An J, et al. Cutting edge: antimalarial drugs inhibit IFN- $\beta$ production through blockade of cyclic GMP-AMP synthase-DNA interaction. J Immunol. 2015;194(9):4089-4093.

197. Gadina M, et al. Translational and clinical advances in JAK-STAT biology: the present and future of jakinibs. J Leukoc Biol. 2018;104(3):499-514.

198. Wallace DJ, et al. Baricitinib for systemic lupus erythematosus: a double-blind, randomised, placebo-controlled, phase 2 trial. Lancet. 2018;392(10143):222-231.

199. Moulton VR, et al. Pathogenesis of human systemic lupus erythematosus: a cellular perspective. Trends Mol Med. 2017;23(7):615-635.

200.Zavada J, et al. Cyclosporine A or intravenous cyclophosphamide for lupus nephritis: the Cyclofa-Lune study. Lupus. 2010;19(11):1281-1289. 